\title{
Suppression of learning about a hard cue by the presence of an easy cue'
}

ELIJAH LOVEJOY 2 AND D. GRAHAM RUSSELL UNIVERSITY OF SUSSEX

Two groups of animals were trained on simultaneous discriminations. On each trial one alternative was vertical, the other horizontal; one was black and the other white. The vertical was rewarded for both groups. For one group, blackwhite was also relevant (and redundant) while for the other black-white was irrelevant. When black-white was relevant, learning about the comparative ly difficult orientation cue was suppressed. Theoretical implications are discussed.

A fundamental assumption of those who postulate attention in discrimination learning (e.g., Sutherland, 1964; Zeaman \& House, 1963; Lovejoy, 1966, 1967) is that speed of learning about a cue depends upon that cue's relative dominance in the population of available cues. On the basis of this assumption, an animal should learn more about a relevant cue $A$ when $A$ is the only visual cue present than when two cues, cue $A$ and cue $B$, are both present and (redundantly) relevant. Babb (1953) has presented data which support this assertion, but as Sutherland \& Andelman (1967) have pointed out, his results may have a simpler explanation: In order to assess learning about cue $A$, after training with $A$ alone or with $A$ and $B$, he tested with A alone. Although he found that the two-cue group performed less well in the test, this may simply have been due to generalization decrement. The twocue group was tested in a changed situation, while the one-cue group continued with the same situation as in training. So Babb's test was inadequate.

In an effort to obviate this objection to Babb's experiment, Sutherland and Andelman trained each of two groups with two cues present, but for one group both were relevant (and redundant) while for the other one cue was irrelevant. Thus each group had been exposed to both cues in training, and generalization decrement in testing should be comparable for the two. It should be noted that this change in design implicates an additional assumption of "attention theory," i.e., that animals attend less to a cue when it is irrelevant than when it is relevant. Put differently, the assumption being tested is now, "Cue A will interfere more with learning about cue $B$ when A is relevant than when A is irrelevant." This is plainly a more complex assumption than the stimulussampling one discussed above.

The results of Sutherland and Andelman's Experiment I are abundantly clear: Animals learn more about the relevant cue A (horizontal-vertical) when cue B (blackwhite) is also relevant than when cue $B$ is irrelevant, in direct contradiction to attention theory.

Two possible reasons for their results may be sug- gested: (1) There is some basic weakness in "attention theory." and this result indicates the need for a fundamental revision. (2) The basic notion that learningabout-X reduces learning-about-Y still holds, butother factors, acting in the opposite direction, had a stronger effect in this experiment.

Sutherland and Andelman opted for the first alternative, and suggested a "hierarchy of stimulus analyzers" as the desired revision. If such a hierarchy exists, we have the situation "learning-about- $X$ may help or hinder learning-about-Y, depending on the location of $X$ and $Y$ in the analyzing hierarchy"-the kind of theoretical position which is hard to disprove.

A case can be made for the second alternative. The factor which may have been acting is the omnipresent one of observing responses; it may be that animals with both cues relevant learned quickly, and so looked at the stimuli on a high proportion of trials. The other group, faced with a more difficult problem, may have looked at the stimuli much less often. As a result, they would have learned less about their sole relevant cue than did the two-cue group. At this point one may wonder what change Sutherland and Andelman could have made in their design to increase the importance of "attention" factors enough to overcome the hypothesized effect of observing factors. An answer is suggested by a formal model for discrimination learning recently proposed by Lovejoy (1967). According to that model, when two cues are relevant a $\mathrm{S}$ will tend to learn first about one of those cues. The extent to which he learns about the second cue depends on how obvious the second cue is; the less obvious the second cue, the less rapidly will learning about it take place. This suggests that Sutherland and Andelman might have obtained the opposite result if they had used one easy cue (relevant for one group, irrelevant for the other) and one difficult cue (always relevant). The present experiment tests this possibility. Method

The Ss were 19 experimentally naive, male, hooded rats, 100 days old. The apparatus was a modified Lashley jumping stand, with landing platforms beneath the stimulus doors (so that no punishment followed an error). For a complete description, see Mackintosh (1963). Animals were first pretrained to jump to oper doors, and then to jump to closed gray doors, behind which they found food. Throughout the experiment, each rat received 10 correction trials per day. After three consecutive wrong choices on a single trial, an animal was guided to the positive stimulus. The stimuli were plexiglas rectangles mounted either vertically 


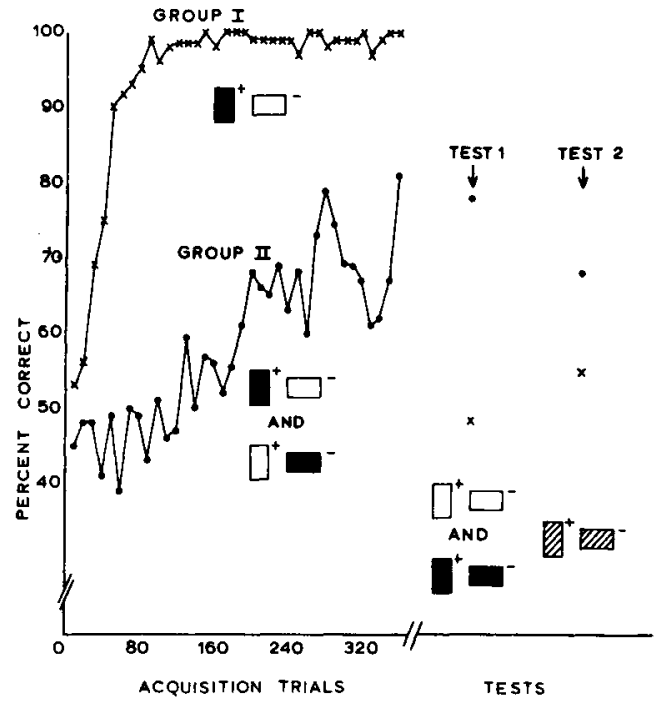

Fig. 1.

or horizontally on the centers of the gray doors of the jumping stand. In order to increase the difficulty of the horizontal-vertical cue, the rectangles were more nearly square $(3.3 \times 6.0 \mathrm{~cm})$ than those used by Sutherland and Andelman $(2.5 \times 10 \mathrm{~cm})$. Black and white rectangles were used during acquisition and Test 1 , and gray rectangles were used during Test 2 . The side of the positive stimulus was determined by Gellermann orders. The stimuli are shown in Fig. 1. Training was continued for 36 days ( 360 trials). After training, two retraining tests were conducted to assess learning about the horizontal-vertical cue.

Results

The entire experiment is summarized in Fig. 1. On the left are the mean learning curves for the two groups. Group 1, with both cues relevant, learned very quickly, and Group 2, with only orientation relevant, learned painfully slowly. This was a very difficult orientation discrimination. The results for the two retraining tests are also shown in Fig. 1, on the right. On the first test Group 1 scored $49 \%$ correct, and Group $278 \%$. This difference is highly significant ( $p<.005$, median test). A second test confirmed this result; Group 1 scored $55 \%$ and Group 2 scored $68 \%$. Again, the difference is significant $(p<.005$, median test). On neither test did the performance of Group 1 differ significantly from chance. Thus the presence of the easy redundant brightness cue seems to have prevented learning from occurring about the difficult orientation cue.

\section{Discussion}

We have demonstrated that learning about a difficult cue is suppressed by the presence of an easy cue, provided that the easy cue is relevant. How does this happen? The relevance of the easy cue is crucial; it seems fair to say that the animal "discovers" this relevance at about the time of solution. Put differently, it seems that once the animal has solved the problem by means of the easy cue, he ceases to learn about the hard cue. There appear to be two alternative mechanisms which might produce such an effect: (1) Once the animal has "solved" the problem, he may fust stop learning altogether. In this case a two-cuesrelevant group has fewer effective trials. (2) The animal may learn predominantly about the dimension he uses to control his behavior-so that once he has solved the problem in terms of the easy cue, he learns primarily about that cue, to the exclusion of others.

Lovejoy (1967) has proposed a formal model with a mechanism of the second type; this model does produce behavior very like that reported above. Animals trained with an easy cue and a hard cue both relevant learn little about the hard cue, whereas animals trained with the same cues present, but only the hard cue relevant, solve the problem.

It is worth noting explicitly that the phenomenon reported here cannot be explained by an "stimulus elements" argument-whereas Babb's results could be. Here the same stimulus elements are presented to both groups, and the difference in learning about orientation must be due to the different correlation of the easy cue with reward.

\section{References}

Babb, H. Transfer from a stimulus complex to differentially disciminable components. J. comp. physiol. Psychol., 1956, 49, 288-291.

Lovejoy, E. Analysis of the overlearning reversal effect. Psychol. Rev., 1966, 73, 87-103.

Lovejoy, E. Attention in discrimination learning. San Francisco: Holden-Day, in press.

Mackintosh, N. J. The effect of irrelevant cues on reversal learning in the rat. Brit. J. Psychol., 1963, 54, 127-134.

Sutherland, N. S. The learning of discriminations by animals. Endeavor, 1964, 23, 148-152.

Sutherland, N. S., \& Andelman, Lani. Learning with one and two cues. Psychon. Sci., 1967, 7, 107-108.

Zeaman, D., \& House, B. J. The role of attention in retardate discrimination learning. In N. R. Ellis (Ed.), Handbook in mental deficiency: Psychological theory and research. New York: McGraw-Hill, 1963. Pp. 159223.

\section{Notes}

1. This work was carried out during the first author's tenure of a North American Treaty Organization Postdoctoral Fellowship in Science, and was supported in part by the American Office of Naval Research, Contract N 62558-4791. We are grateful to $M$. S, Halliday and N. S. Sutherland, who commented on the manuscript. 2. Now at the University of California, Santa Barbara. 